\title{
General solution of a fractional diffusion-advection equation for solar cosmic-ray transport
}

\author{
M.C. Rocca ${ }^{\mathrm{a}, \mathrm{b}, *}$, A.R. Plastino ${ }^{\mathrm{c}, \mathrm{d}}$, A. Plastino ${ }^{\mathrm{a}, \mathrm{b}}$, G.L. Ferri ${ }^{\mathrm{e}}$, A. de Paoli ${ }^{\mathrm{a}, \mathrm{b}}$ \\ a Departamento de Física, Fac. de Ciencias Exactas, Universidad Nacional de La Plata, C.C. 67 (1900) La Plata, Argentina \\ ${ }^{\mathrm{b}}$ IFLP-CCT-CONICET, C.C 727 (1900) La Plata, Argentina \\ ${ }^{c}$ CeBio y Secretaria de Investigacion, Univ. Nac. del Noroeste de la Prov. de Bs. As., UNNOBA, R. Saenz Peña 456, Junin, Argentina \\ d CONICET, R. Saenz Peña 456, Junin, Argentina \\ e Faculty of Exact and Natural Sciences, La Pampa National University, Uruguay 151, Santa Rosa, La Pampa, Argentina
}

\section{H I G H L I G H T S}

- We solve the fractional diffusion-advection equation for solar cosmic-ray transport.

- We give its general solution.

- A numerical analysis of this equation is made.

- We use hypergeometric distributions.

\section{A R T I C L E I N F O}

\section{Article history:}

Received 30 August 2015

Received in revised form 6 December 2015

Available online 24 December 2015

\section{A B S T R A C T}

In this effort we exactly solve the fractional diffusion-advection equation for solar cosmicray transport and give its general solution in terms of hypergeometric distributions. Numerical analysis of this equation shows that its solutions resemble power-laws.

(C) 2015 Elsevier B.V. All rights reserved.

\section{Keywords:}

Distributions

Cosmic rays

Diffusion

Sun

Heliosphere

\section{Introduction}

There is a considerable body of evidence, from data collected by spacecrafts like Ulysses and Voyager 2, indicating that the transport of energetic particles in the turbulent heliospheric medium is superdiffusive [1,2]. Considerable effort has been devoted in recent years to the development of superdiffusive models for the transport of electrons and protons in the heliosphere [3-5]. This kind of transport regime exhibits a power-law growth of the mean square displacement of the diffusing particles, $\left\langle\Delta x^{2}\right\rangle \propto t^{\alpha}$, with $\alpha>1$ (see, for instance, Ref. [6]). The special case $\alpha=2$ is called ballistic transport. The limit case $\alpha \rightarrow 1$ corresponds to normal diffusion, described by the well-known Gaussian propagator. The energetic particles detected by the aforementioned probes are usually associated with violent solar events like solar flares. These particles diffuse in the solar wind, which is a turbulent environment than can be assumed statistically homogeneous at

\footnotetext{
* Corresponding author at: Departamento de Física, Fac. de Ciencias Exactas, Universidad Nacional de La Plata, C.C. 67 (1900) La Plata, Argentina.

E-mail address: mariocarlosrocca@gmail.com (M.C. Rocca).
} 
large enough distances from the sun [1]. This implies that the propagator $P\left(x, x^{\prime}, t, t^{\prime}\right)$, describing the probability of finding at the space time location $(x, t)$ a particle that has been injected at $\left(x^{\prime}, t^{\prime}\right)$, depends solely on the differences $x-x^{\prime}$ and $t-t^{\prime}$. In the superdiffusive regime the propagator $P\left(x, x^{\prime}, t, t^{\prime}\right)$ is not Gaussian, and exhibits power-law tails. It arises as solution a non local diffusive process governed by an integral equation that can be recast under the guise of a diffusion equation where the well-known Laplacian term is replaced by a term involving fractional derivatives [7]. Diffusion equations with fractional derivatives have attracted considerable attention recently (see Refs. [8-12] and references therein) and have lots of potential applications $[13,14]$. In particular, the observed distributions of solar cosmic ray particles are often consistent with power-law tails, suggesting that a superdiffusive process is at work.

A proper understanding of the transport of energetic particles in space is a vital ingredient for the analysis of various important phenomena, such as the propagation of particles from the Sun to our planet or, more generally, the acceleration and transport of cosmic rays. The superdiffusion of particles in interplanetary turbulent environments is often modeled using asymptotic expressions for the pertinent non-Gaussian propagator, which have a limited range of validity. A first step towards a more accurate analytical treatment of this problem is to consider solutions of a fractional diffusion-advection equation describing the diffusion of particles emitted at a shock front that propagates at a constant upstream speed $V_{s h}$ in the solar wind rest frame. The shock front is assumed to be planar, leading to an effectively one-dimensional problem. Each physical quantity depends only on the time $t$ and on the spatial coordinate $x$ measured along an axis perpendicular to the shock front.

There are many ways of evaluating fractional derivatives of either functions or distributions.

(1) Defining the derivative using ultradistributions à la Sebastiao e Silva, as done in Ref. [15].

(2) Using Caputo's fractional derivatives, Riemann-Liouville ones, etc.

In the present contribution we re-visit the fractional diffusion-advection equation previously studied in other papers (see Ref. [15] and references therein). Why? Because we were able to give it a simplified treatment, clearly accessible to the entire scientific community, unlike that of Ref. [15], that appeals to ultradistributions of exponential type, a very sophisticated topic, accessible only to people familiar with extremely complex mathematics.

\section{Formulation of the problem}

Consider the equation

$$
\frac{\partial f}{\partial t}=\kappa \frac{\partial^{\alpha} f}{\partial|x|^{\alpha}}+a \frac{\partial f}{\partial x}+\delta(x),
$$

where $t>0$ and $f(x, t)$ is the distribution function for solar cosmic-rays transport. Here the fractional spatial derivative is defined as

$$
\frac{\partial^{\alpha} f}{\partial|x|^{\alpha}}=\frac{1}{\pi} \sin \left(\frac{\pi \alpha}{2}\right) \Gamma(\alpha+1) \int_{0}^{\infty} \frac{f(x+\xi)-2 f(x)+f(x-\xi)}{\xi^{\alpha+1}} \mathrm{~d} \xi .
$$

To solve this equation we use the Green function governed by the equation:

$$
\frac{\partial g}{\partial t}=\kappa \frac{\partial^{\alpha} g}{\partial|x|^{\alpha}}+\delta(x) \delta(t)
$$

With this Green function, the solution of (2.1) can be expressed as

$$
f(x, t)=\int_{0}^{t} g\left(x+a t^{\prime}, t^{\prime}\right) \mathrm{d} t^{\prime} .
$$

In this work we obtain the solutions of Eqs. (2.1) and (2.3) using distributions as main tools [16].

For our task we use, as a first step, $t$ the Green function through the use of the Fourier Transform given by

$$
\hat{g}(k, t)=\frac{1}{2 \pi} \int_{-\infty}^{\infty} g(x, t) \mathrm{e}^{-\mathrm{i} k x} \mathrm{~d} x,
$$

from which we obtain for $\hat{g}$ :

$$
\hat{g}(k, t)=-\kappa|k|^{\alpha} \hat{g}(k, t)+\frac{1}{2 \pi} \delta(t),
$$

whose solution is

$$
\hat{g}(k, t)=\frac{H(t)}{2 \pi} \mathrm{e}^{-\kappa|k|^{\alpha} t},
$$

where $H(t)$ is Heaviside's step function. 


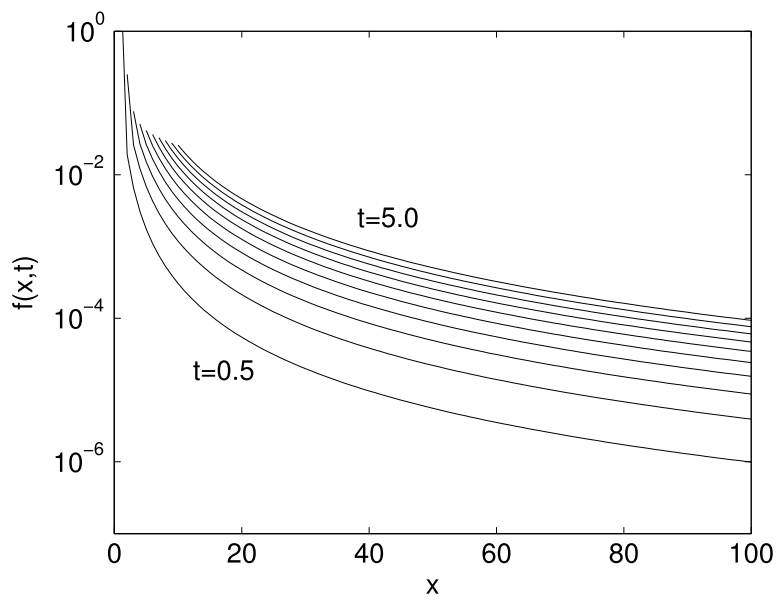

Fig. 1. Typical graph for solutions of Eq. (3.5), for different times $t$ ( $x$ in arbitrary units).

\section{General solution of the equations}

From (2.7) we have for $\hat{g}$

$$
\hat{g}(k, t)=\frac{H(t)}{2 \pi} \mathrm{e}^{-\kappa|k|^{\alpha} t}=\frac{H(t)}{2 \pi} \sum_{n=0}^{\infty} \frac{(-1)^{n} \kappa^{n} k^{\alpha n} t^{n}}{n !},
$$

and, invoking the inverse Fourier transform

$$
g(x, t)=\frac{H(t)}{2 \pi} \int_{-\infty}^{\infty} \mathrm{e}^{-\kappa|k|^{\alpha} t} \mathrm{e}^{\mathrm{i} k x} \mathrm{~d} k=\frac{H(t)}{2 \pi} \sum_{n=0}^{\infty} \frac{(-1)^{n} \kappa^{n} t^{n}}{n !}\left[\int_{0}^{\infty} k^{\alpha n} \mathrm{e}^{\mathrm{i} k x} \mathrm{~d} x+\int_{0}^{\infty} k^{\alpha n} \mathrm{e}^{-\mathrm{i} k x} \mathrm{~d} x\right] .
$$

Fortunately, we can find in the classical book of Ref. [16] the results for the two integrals of (3.2). We obtain

$$
g(x, t)=\frac{H(t)}{2 \pi} \sum_{n=0}^{\infty} \frac{(-1)^{n} \kappa^{n} t^{n}}{n !} \Gamma(\alpha n+1)\left[\frac{\mathrm{e}^{\mathrm{i} \frac{\pi}{2}(\alpha n+1)}}{(x+\mathrm{i} 0)^{\alpha n+1}}+\frac{\mathrm{e}^{-\mathrm{i} \frac{\pi}{2}(\alpha n+1)}}{(x-\mathrm{i} 0)^{\alpha n+1}}\right] .
$$

Using now (2.4) we have for $f$

$$
f(x, t)=\int_{0}^{t} g\left(x+a t^{\prime}, t^{\prime}\right) \mathrm{d} t^{\prime},
$$

so that one can write

$$
f(x, t)=\frac{1}{2 \pi} \sum_{n=0}^{\infty} \frac{(-1)^{n} \kappa^{n}}{n !} \Gamma(\alpha n+1) \int_{0}^{t}\left[\frac{\mathrm{e}^{\mathrm{i} \frac{\pi}{2}(\alpha n+1)}}{\left(x+a t^{\prime}+\mathrm{i} 0\right)^{\alpha n+1}}+\frac{\mathrm{e}^{-\mathrm{i} \frac{\pi}{2}(\alpha n+1)}}{\left(x+a t^{\prime}-\mathrm{i} 0\right)^{\alpha n+1}}\right] t^{\prime n} \mathrm{~d} t^{\prime} .
$$

According to Eq. (A.1) of the Appendix, where $t>0$, we now obtain for $f$, invoking hypergeometric functions $F(\alpha n+$ $1,2 ; 3 ; z)$ and Beta functions $\mathscr{B}(1, n+1)$,

$$
\begin{aligned}
f(x, t)= & \frac{1}{2 \pi} \sum_{n=0}^{\infty} \frac{(-1)^{n} \kappa^{n} t^{n+1}}{n !} \Gamma(\alpha n+1) \mathcal{B}(1, n+1)\left[\frac{\mathrm{e}^{\mathrm{i} \frac{\pi}{2}(\alpha n+1)}}{(x+\mathrm{i} 0)^{\alpha n+1}} F\left(\alpha n+1, n+1 ; n+2 ;-\frac{a t}{x+\mathrm{i} 0}\right)\right. \\
& \left.+\frac{\mathrm{e}^{-\mathrm{i} \frac{\pi}{2}(\alpha n+1)}}{(x-\mathrm{i} 0)^{\alpha n+1}} F\left(\alpha n+1, n+1 ; n+2 ;-\frac{a t}{x-\mathrm{i} 0}\right)\right] .
\end{aligned}
$$

This is the general solution of Eq. (2.1) for the initial condition $f(x, 0)=0$.

Fig. 1 depicts a typical graph for solutions of Eq. (3.5). We do this for different times $t$. The main result of this contribution is that these curves are very well approximated by power laws. Fig. $2(\kappa=0.01), 3(\kappa=0.5)$, and $4(\kappa=0.2)$ show how the above solutions can be adjusted by power laws of the form $1 / x^{q}$ : the corresponding lines rotate, for fixed $\alpha=3 / 2$, in the plane $f(x, t)$ vs. $1 / x^{q}$ when at changes, as the plots clearly illustrate. In all our figures we have taken $a=1$ and $\alpha=3 / 2$ (see Figs. 3 and 4 ).

Fig. 5 shows how the dependence of $q$ with $\alpha$ can, in turn, be adjusted in simple fashion. In Figs. 5 and 6, straight lines are employed. It is evident that a weak diffusion-regime exists. This regime was discussed in Ref. [15] and references therein. 


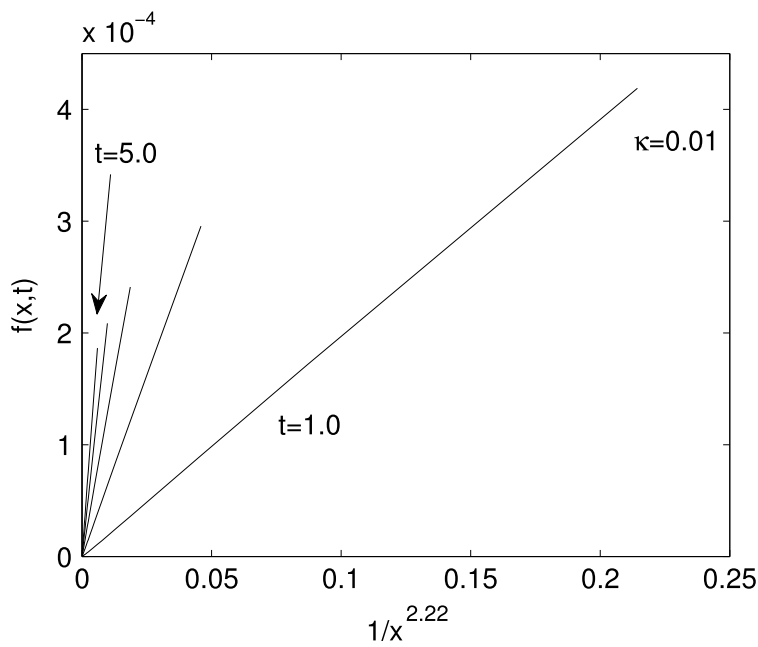

Fig. 2. Power-law fitting to the solutions of Eq. (3.5) for $\alpha=3 / 2 a=1$, and $\kappa=0.01$, at different times $t$.

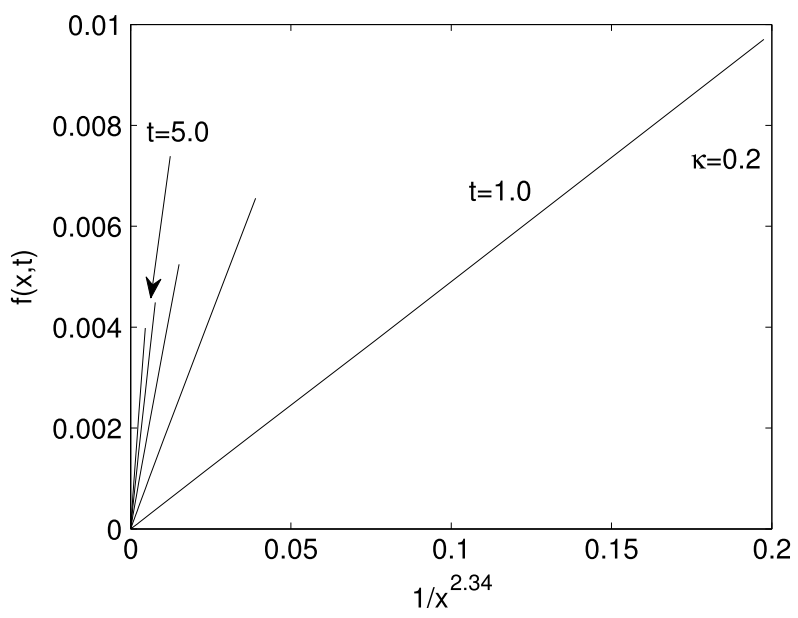

Fig. 3. Power-law fitting to the solutions of Eq. (3.5) for $\alpha=3 / 2 a=1$, and $\kappa=0.5$, at different times $t$.

Here we not only confirm its existence via our exact approach, but also show that it extends up to rather significant $\kappa$-values of the order of 0.5 .

What about other $\alpha$ values? The situation does not change. Power-law adjustment continues to be possible. Figs. 6 and 7 illustrate this issue.

\section{Weak diffusion approximation}

We shall now consider a weak diffusion approximation. Within this approximation we can treat $\kappa$ as a small parameter and develop $f$ up to order one ([15] and references therein). Thus, we can write:

$$
f(x, t)=f_{0}(x, t)+f_{1}(x, t),
$$

where (i) $f_{0}$ is independent of $\kappa$ and (ii) in $f_{1}$ the corresponding power of $\kappa$ is unity.

Eq. (3.5) entails that we have, for $f_{0}(n=0$ in (3.5)),

$$
f_{0}(x, t)=\frac{i t}{2 \pi}\left[(x+\mathrm{i} 0)^{-1} F\left(1,1 ; 2 ;-\frac{a t}{x+\mathrm{i} 0}\right)-(x-\mathrm{i} 0)^{-1} F\left(1,1 ; 2 ;-\frac{a t}{x-\mathrm{i} 0}\right)\right] .
$$

Recourse to the celebrated tables of Ref. [17] allows us to write

$$
F(1,1 ; 2 ;-z)=\frac{1}{z} \ln (1+z),
$$




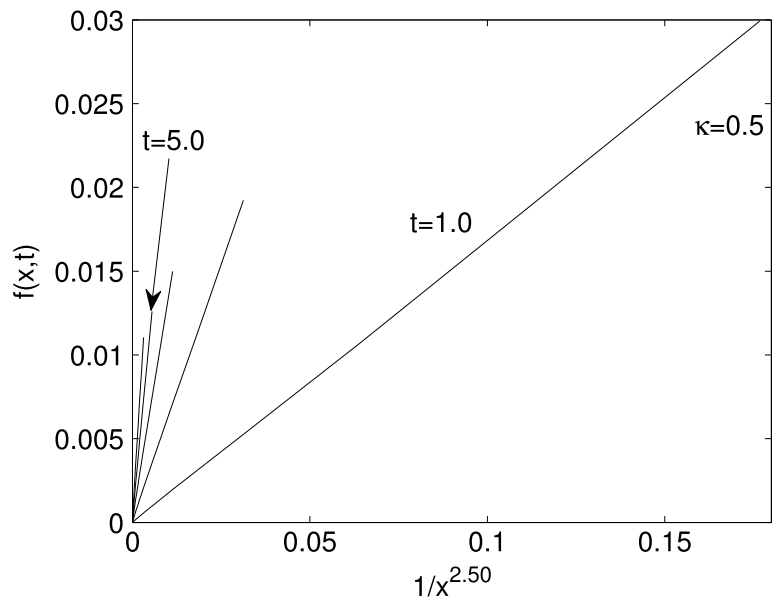

Fig. 4. Power-law fitting to the solutions of Eq. (3.5) for $\alpha=3 / 2, a=1$, and $\kappa=0.2$, at different times $t$.

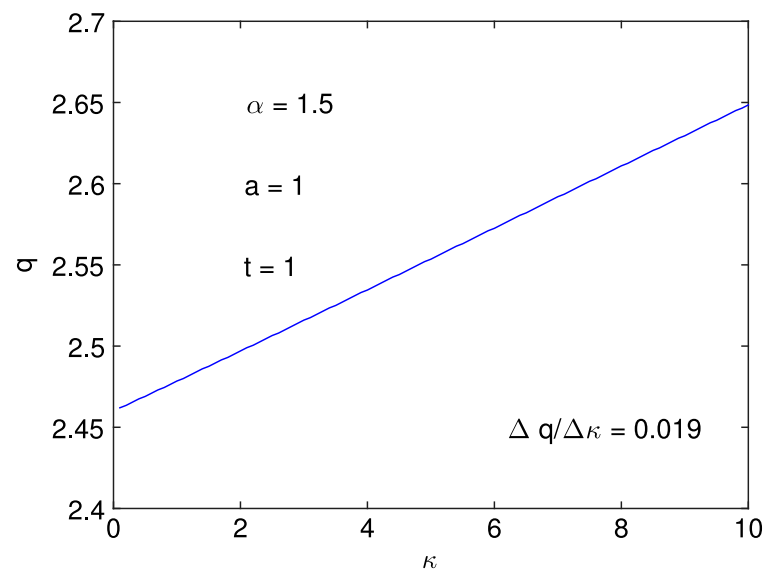

Fig. 5. Possible linear relationship between the power-law exponent $q$ and $\kappa$.

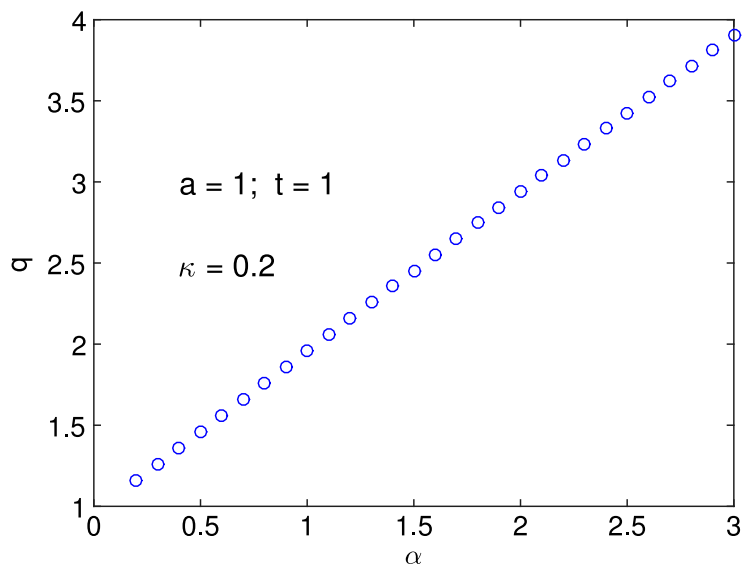

Fig. 6. Fractional derivative of order $\alpha$ vs. power law exponent $q$, fitting the solutions of the fractional advection-diffusion equation $(\kappa=0.2)$.

and we obtain for $f_{0}$

$$
f_{0}(x, t)=\frac{1}{a}[H(-x)-H(-x-a t)]=\frac{1}{2 a}[\operatorname{Sgn}(x+a t)-\operatorname{Sgn}(x)] .
$$




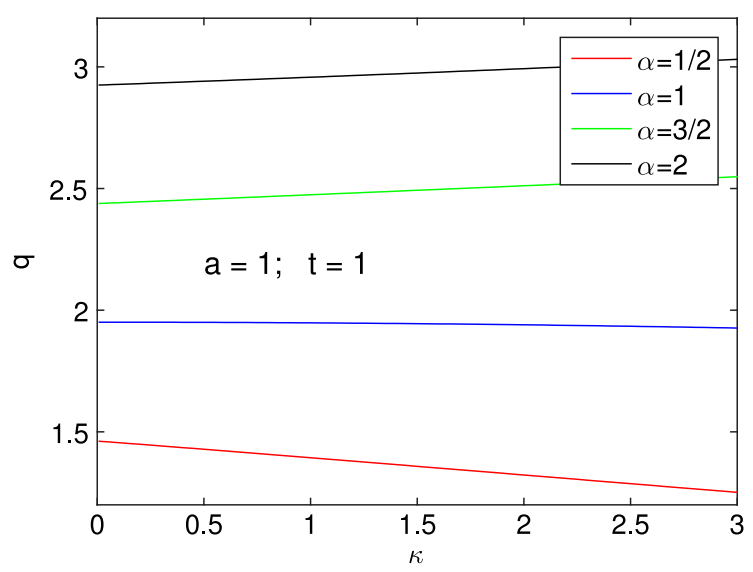

Fig. 7. $q$ vs. $\kappa$ for different $\alpha$-values.

When we take $n=1$ in (3.5), $f_{1}$ is defined as

$$
\begin{aligned}
f_{1}(x, t)= & -\frac{\mathrm{i} \kappa t^{2}}{4 \pi} \Gamma(\alpha+1)\left[\frac{\mathrm{e}^{\mathrm{i} \frac{\pi}{2}}}{(x+\mathrm{i} 0)^{\alpha+1}} F\left(\alpha+1,2 ; 3 ;-\frac{a t}{x+\mathrm{i} 0}\right)\right. \\
& \left.+\frac{\mathrm{e}^{-\mathrm{i} \frac{\pi}{2}}}{(x-\mathrm{i} 0)^{\alpha+1}} F\left(\alpha+1,2 ; 3 ;-\frac{a t}{x-\mathrm{i} 0}\right)\right] .
\end{aligned}
$$

Now, from (A.1) of Appendix we have, for the hypergeometric function,

$$
F(\alpha+1,2 ; 3 ; z)=\frac{2}{\alpha(\alpha-1) z^{2}}\left[1+\frac{\alpha z-1}{(1-z)^{\alpha}}\right],
$$

so that, using this result, $f_{1}$ adopts the form

$$
f_{1}(x, t)=\frac{\mathrm{i} \kappa \Gamma(\alpha-1)}{2 \pi a^{2}}\left\{(x+\alpha a t)\left[\frac{\mathrm{e}^{\mathrm{i} \frac{\pi}{2} \alpha}}{(x+a t+\mathrm{i} 0)^{\alpha}}-\frac{\mathrm{e}^{-\mathrm{i} \frac{\pi}{2} \alpha}}{(x+a t-\mathrm{i} 0)^{\alpha}}\right]+\frac{\mathrm{e}^{-\mathrm{i} \frac{\pi}{2} \alpha}}{(x-\mathrm{i} 0)^{\alpha-1}}-\frac{\mathrm{e}^{\mathrm{i} \frac{\pi}{2} \alpha}}{(x+\mathrm{i} 0)^{\alpha-1}}\right\} .
$$

Using at this point (4.1), (4.4), and (4.6), the final result for $f$, up to first order in $\kappa$, reads, invoking the sign function $\operatorname{Sgn}(x)$,

$$
\begin{aligned}
f(x, t)= & \frac{1}{2 a}[\operatorname{Sgn}(x+a t)-\operatorname{Sgn}(x)]+\frac{\mathrm{i} \kappa \Gamma(\alpha-1)}{2 \pi a^{2}}\left\{(x+\alpha a t)\left[\frac{\mathrm{e}^{\mathrm{i} \frac{\pi}{2} \alpha}}{(x+a t+\mathrm{i} 0)^{\alpha}}-\frac{\mathrm{e}^{-\mathrm{i} \frac{\pi}{2} \alpha}}{(x+a t-\mathrm{i} 0)^{\alpha}}\right]\right. \\
& \left.+\frac{\mathrm{e}^{-\mathrm{i} \frac{\pi}{2} \alpha}}{(x-\mathrm{i} 0)^{\alpha-1}}-\frac{\mathrm{e}^{\mathrm{i} \frac{\pi}{2} \alpha}}{(x+\mathrm{i} 0)^{\alpha-1}}\right\} .
\end{aligned}
$$

Thus, for $x>0$ (4.7) becomes

$$
f(x, t)=\frac{\kappa \sin \left(\frac{\pi \alpha}{2}\right) \Gamma(\alpha-1)}{\pi a^{2}}\left[\frac{1}{x^{\alpha-1}}-\frac{x+\alpha a t}{(x+a t)^{\alpha}}\right] .
$$

We have to distinguish two limiting cases. The first one is the asymptotic situation $x \gg a t$. In this case,

$$
f(x, t)=\frac{1}{2 \pi} \sin \left(\frac{\pi \alpha}{2}\right) \Gamma(\alpha+1) \frac{\kappa t^{2}}{\chi^{\alpha+1}} .
$$

The second limiting case is $0<x \ll a t$. The corresponding expression for $f$ becomes

$$
f(x, t)=\frac{1}{\pi} \sin \left(\frac{\pi \alpha}{2}\right) \Gamma(\alpha-1) \frac{\kappa}{a^{2}} x^{1-\alpha} .
$$

We consider signs now. When $x+a t<0$, from (4.7) we have

$$
f(x, t)=\frac{\kappa \sin \left(\frac{\pi \alpha}{2}\right) \Gamma(\alpha-1)}{\pi a^{2}}\left[\frac{1}{|x|^{\alpha-1}}+\frac{x+\alpha a t}{|x+a t|^{\alpha}}\right] .
$$




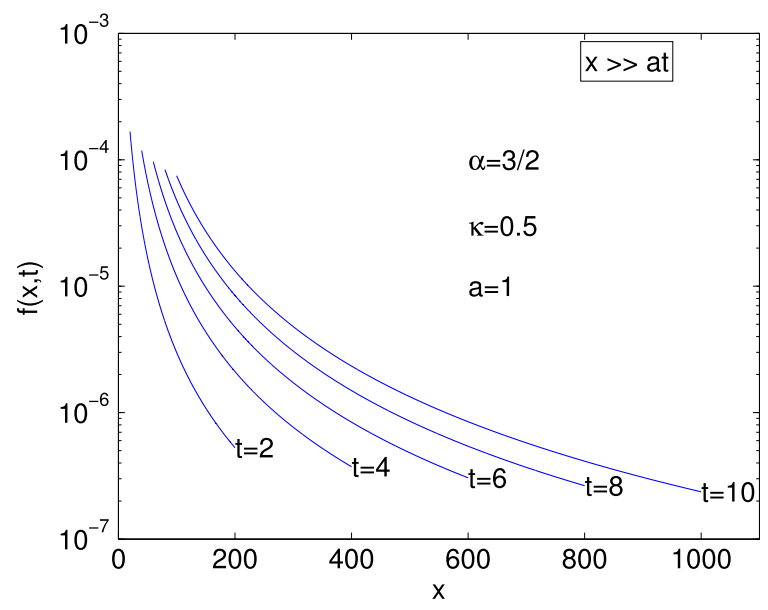

Fig. 8. $x \gg$ at: Plot of $f(x, t)$ vs. $x$ for $a=1, \alpha=3 / 2$, and $\kappa=0.5$.

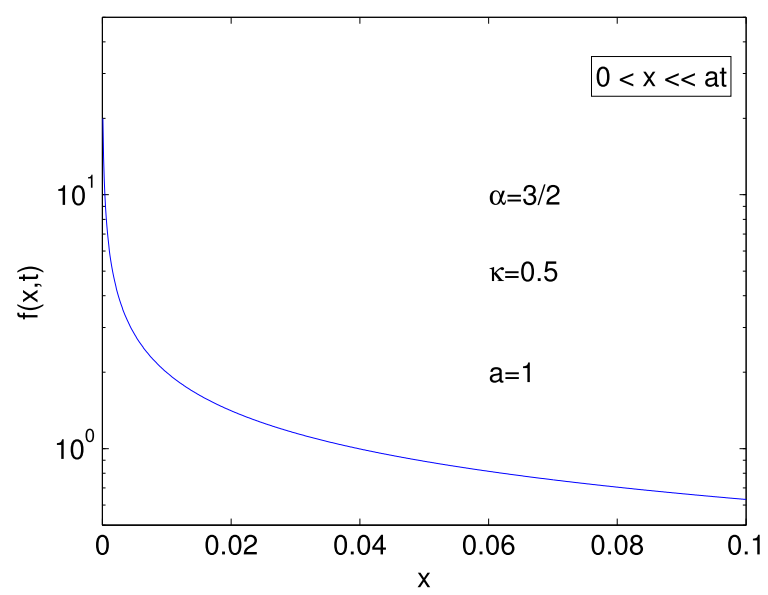

Fig. 9. $0<x \ll$ at: Plot of $f(x, t)$ vs. $x$ for $a=1, \alpha=3 / 2$, and $\kappa=0.5$.

Again, two special cases must be considered. One is for $x \ll-a t$ for which

$$
f(x, t)=\frac{1}{2 \pi} \sin \left(\frac{\pi \alpha}{2}\right) \Gamma(\alpha+1) \frac{\kappa t^{2}}{|x|^{\alpha+1}} .
$$

The other special situation is $x<0, x+a t>0, x \gg-a t$. Here (from (4.7) again),

$$
f(x, t)=\frac{1}{a}+\frac{1}{\pi} \sin \left(\frac{\pi \alpha}{2}\right) \Gamma(\alpha-1) \frac{\kappa}{a^{2}}|x|^{1-\alpha} .
$$

We can see below graphs corresponding to Eqs. (4.9), (4.10), (4.12), and (4.13). Note the similarity between these graphs and the graph displayed in Fig. 1, that represents the general solution. This fact confirms our assertion about the power-law behavior of the general solution (see Figs. 8-11).

\section{Change of variables}

We assume that, in the solar wind's rest frame, the particles' transport is described by the fractional-diffusion equation with no advection term (that is, with $a=0$ in (2.1)). The shock front (which started at $x_{0}=-V_{s h} t_{0}$, moves with constant speed $V_{s h}$, and is regarded as highly localized in the $x$-coordinate) constitutes the source of the particles. Consequently, we have a fractional-diffusion equation with a uniformly moving Dirac's delta source of the form $\delta\left(x-V_{s h} t\right)$. In order to have a stationary delta source we need to perform an appropriate change of coordinates, re-casting our problem in a reference frame for which the shock front is stationary. We also change the origin of time so that the source starts being active at $t=0$. In this new reference frame the transport equation has an advection term with advection velocity $a=V_{s h}$, and a stationary source $\delta(0)$ that starts at $t=0$. After solving the diffusion-advection equation in this new frame (which is what we have done in the previous sections), we re-express the solution in terms of the original coordinates associated with the solar wind 


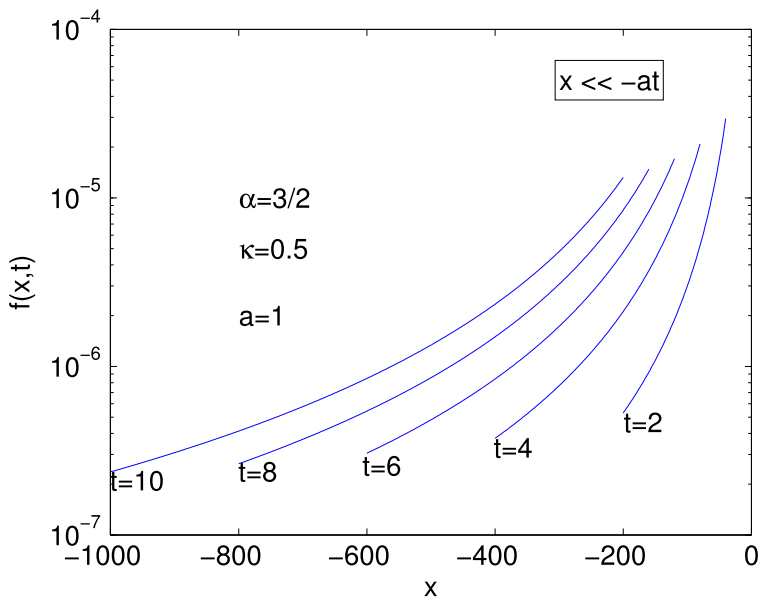

Fig. 10. $x \ll-a$ : Plot of $f(x, t)$ vs. $x$ for $a=1, \alpha=3 / 2$, and $\kappa=0.5$.

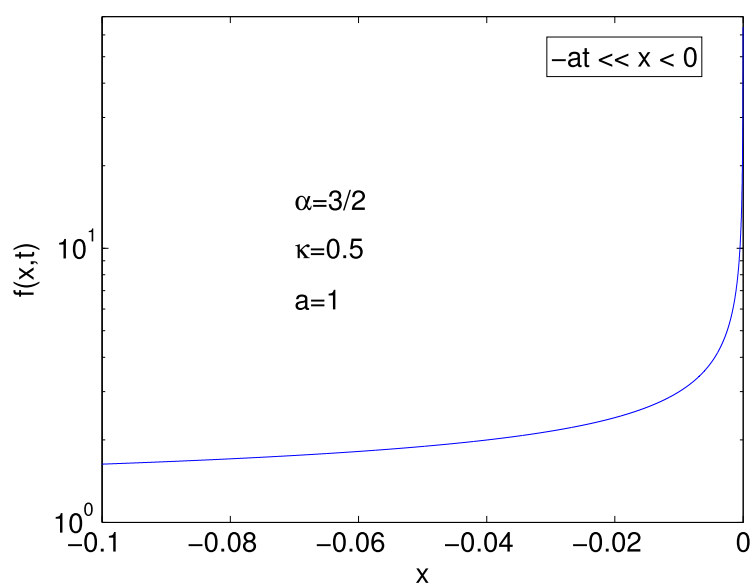

Fig. 11. $-a t \ll x<0$ : Plot of $f(x, t)$ vs. $x$ for $a=1, \alpha=3 / 2$, and $\kappa=0.5$.

rest frame. This last step is succinctly described by the three correspondences $a \rightarrow v_{\text {sh }}, t \rightarrow t+t_{0}$, and $x \rightarrow x-v_{\text {sh }} t$, after which Eq. (3.5) adopts the appearance

$$
\begin{aligned}
f(x, t)= & \frac{1}{2 \pi} \sum_{n=0}^{\infty} \frac{(-1)^{n} \kappa^{n}\left(t+t_{0}\right)^{n+1}}{n !} \Gamma(\alpha n+1) B(1, n+1) \\
& \times\left[\frac{\mathrm{e}^{\mathrm{i} \frac{\pi}{2}(\alpha n+1)}}{\left(x-v_{s h} t+\mathrm{i} 0\right)^{\alpha n+1}} F\left(\alpha n+1, n+1 ; n+2 ;-\frac{v_{s h}\left(t+t_{0}\right)}{x-v_{s h} t+\mathrm{i} 0}\right)\right. \\
& \left.+\frac{\mathrm{e}^{-\mathrm{i} \frac{\pi}{2}(\alpha n+1)}}{\left(x-v_{s h} t-\mathrm{i} 0\right)^{\alpha n+1}} F\left(\alpha n+1, n+1 ; n+2 ;-\frac{v_{s h}\left(t+t_{0}\right)}{x-v_{s h} t-\mathrm{i} 0}\right)\right] .
\end{aligned}
$$

Accordingly, in the weak diffusion approximation (4.7) we have

$$
\begin{aligned}
f(x, t)= & \frac{1}{2 v_{s h}}\left[\operatorname{Sgn}\left(x+v_{s h} t_{0}\right)-\operatorname{Sgn}\left(x-v_{s h} t\right)\right]+\frac{\mathrm{i} \kappa \Gamma(\alpha-1)}{2 \pi V_{s h}^{2}}\left\{( x + ( \alpha - 1 ) v _ { s h } t + v _ { s h } t _ { 0 } ) \left[\frac{\mathrm{e}^{\mathrm{i} \frac{\pi}{2} \alpha}}{\left(x+v_{s h} t_{0}+\mathrm{i} 0\right)^{\alpha}}\right.\right. \\
& \left.\left.-\frac{\mathrm{e}^{-\mathrm{i} \frac{\pi}{2} \alpha}}{\left(x+v_{s h} t_{0}-\mathrm{i} 0\right)^{\alpha}}\right]+\frac{\mathrm{e}^{-\mathrm{i} \frac{\pi}{2} \alpha}}{\left(x-v_{s h} t-\mathrm{i} 0\right)^{\alpha-1}}-\frac{\mathrm{e}^{\mathrm{i} \frac{\pi}{2} \alpha}}{\left(x-v_{s h} t+\mathrm{i} 0\right)^{\alpha-1}}\right\} .
\end{aligned}
$$




\section{Conclusions}

We have provided an explicit analytical solution for and advection-diffusion equation involving fractional derivatives in the diffusion term. This equation governs the diffusion of particles in the solar wind injected at the front of a shock that travels at a constant upstream speed $V_{s h}$ in the solar wind rest frame. The shock is assumed to have a planar front, leading to a problem with an effective one dimensional geometry, where all the relevant quantities depend solely on time and on the coordinate $x$ measured along an axis perpendicular to the front.

We obtained the exact solution of the above mentioned equation in the $x$-configuration space (besides the associated formal solution in the $k$-space related to the previous one via a Fourier transform).

We conclude from our analysis that the solutions of the fractional diffusion-advection equations are essentially power laws, and have numerically found a "law" for the behavior of the associated power-exponent $q$ with varying $\kappa$, via spline interpolation.

\section{Appendix. Some properties of hypergeometric function}

Using data from Ref. [18] we have

$$
\int_{0}^{t} \frac{t^{\prime n}}{\left(x+a t^{\prime} \pm \mathrm{i} 0\right)^{\alpha n+1}} \mathrm{~d} t^{\prime}=\frac{t^{n+1}}{(x \pm \mathrm{i} 0)^{\alpha n+1}} B(1, n+1) F\left(\alpha n+1, n+1, n+2 ;-\frac{a t}{x \pm \mathrm{i} 0}\right) .
$$

Now we appeal to the transformation formula given in Ref. [19] for the hypergeometric function

$$
F(\alpha+1,2 ; 3 ; z)=\frac{2 \Gamma(1-\alpha)}{\Gamma(2-\alpha)}(-1)^{\alpha+1} z^{-\alpha-1} F\left(\alpha+1, \alpha-1 ; \alpha ; \frac{1}{z}\right)+\frac{2 \Gamma(\alpha-1)}{\Gamma(\alpha+1)} z^{-2} F\left(2,0 ; 2-\alpha ; \frac{1}{z}\right),
$$

with

$$
F(a, 0 ; c ; z)=1 .
$$

In this circumstance we obtain

$$
F(\alpha+1,2 ; 3 ; z)=\frac{2 \Gamma(1-\alpha)}{\Gamma(2-\alpha)}(-1)^{\alpha+1} z^{-\alpha-1} F\left(\alpha+1, \alpha-1 ; \alpha ; \frac{1}{z}\right)+\frac{2 \Gamma(\alpha-1)}{\Gamma(\alpha+1)} z^{-2} .
$$

Now we invoke the transformation formula [20]

$$
F\left(\alpha+1, \alpha-1 ; \alpha ; \frac{1}{z}\right)=\left(1-\frac{1}{z}\right)^{-\alpha} F\left(-1,1 ; \alpha ; \frac{1}{z}\right),
$$

or

$$
F\left(\alpha+1, \alpha-1 ; \alpha ; \frac{1}{z}\right)=\frac{z^{\alpha}}{(z-1)^{\alpha}}\left(\frac{\alpha z-1}{\alpha z}\right) .
$$

At this stage, we have, finally,

$$
F(\alpha+1,2 ; 3 ; z)=\frac{2}{\alpha(\alpha-1) z^{2}}\left[1+\frac{\alpha z-1}{(1-z)^{\alpha}}\right] .
$$

\section{References}

[1] S. Perri, G. Zimbardo, Astrophys. J. 671 (2007) L000.

2] S. Perri, G. Zimbardo, Astrophys. J. 693 (2009) L118.

[3] T. Sugiyama, D. Shiota, Astrophys. J. 731 (2011) L34.

[4] E.M. Trotta, G. Zimbardo, A\&A 530 (2011) A130.

[5] G. Zimbardo, S. Perri, Astrophys. J. 778 (2013) 35.

[6] A.I. Saichev, G.M. Zaslavsky, Chaos 7 (1997) 753.

[7] K.V. Chukbar, Sov. J. Exp. Theor. Phys. 81 (1995) 1025.

[8] E.K. Lenzi, A.A. Tateishi, H.V. Ribeiro, M.K. Lenzi, G. Gonsalves, L.R. da Silva, J. Stat. Mech. Theory Exp. 8 (2014) 08019

[9] E.K. Lenzi, L.R. da Silva, A.T. Silva, L.R. Evangelista, M.K. Lenzi, Physica A 388 (2009) 806.

[10] R. Rossato, M.K. Lenzi, L.R. Evangelista, E.K. Lenzi, Phys. Rev. E 76 (2007) 032102.

111] R. Stern, F. Effenberger, H. Fichtner, T. Schäfer, Fract. Calc. Appl. Anal. 17 (2014) 171.

[12] E.K. Lenzi, R.S. Mendes, J.S. Andrade Jr., L.R. da Silva, L.S. Lucena, Phys. Rev. E 71 (2005) 052101

[13] R. Metzler, J. Klafter, Phys. Rep. 339 (2000) 1.

[14] D. Perrone, et al., Space Sci. Rev. 178 (2013) 233.

[15] M.C. Rocca, A.R. Plastino, A. PLastino, G.L. Ferri, A.L. De Paoli, J. Stat. Phys. 161 (2015) 986.

[16] I.M. Guelfand, G.E. Chilov, Les Distributions, vol. V1, Dunod, 1962.

[17] I.S. Gradshteyn, I.M. Ryzhik, Table of Integrals, Series and Products, vol. 9.121, Academic Press, 1965, p. 1041, 6.

[18] I.S. Gradshteyn, I.M. Ryzhik, Table of Integrals, Series and Products, vol. 3.197, Academic Press, 1965, p. 287, 8.

[19] I.S. Gradshteyn, I.M. Ryzhik, Table of Integrals, Series and Products, vol. 9.132, Academic Press, 1965, p. 1043, 2

[20] I.S. Gradshteyn, I.M. Ryzhik, Table of Integrals, Series and Products, vol. 9.131, Academic Press, 1965, p. 1043, 1. 